IFUP-TH 13/2001

\title{
Local Fields on the Brane Induced by Nonlocal Fields in the Bulk
}

\author{
Mihail Mintchev \\ Istituto Nazionale di Fisica Nucleare, Sezione di Pisa \\ Dipartimento di Fisica dell'Università di Pisa, \\ Via Buonarroti 2, 56127 Pisa, Italy
}

\begin{abstract}
We investigate quantum field theory in a bulk space with boundary, which represents a 3-brane. Both flat and anti-de Sitter backgrounds are considered. The basic idea is to keep local commutativity only on the brane, giving up this requirement in the bulk. We explore the consequences of this proposal, constructing a large family of nonlocal bulk fields, whose brane relatives are local. We estimate the ultraviolet behavior of these local brane fields, characterizing a subfamily which generates renormalizable theories on the brane. The issue of brane conformal invariance and the relation between bulk and brane conserved currents are also examined in this framework.
\end{abstract}

PACS numbers: 04.50.+h, 11.10.Kk, 11.27.+d, 11.40.-q,

Keywords: quantum fields, branes, AdS space-time, local commutativity.

March 2001 


\section{Introduction}

Triggered by the fast development of brane theory (cf. [1] and references therein) and the revival [2, 3, 田, 5, 6] of the attractive idea [7] to consider the observable universe as a three-brane embedded in a space with more (non)compact dimensions, there is recently great interest in quantum field theory with extra space dimensions. The intriguing fact that strongly motivates the investigation of such a scenario is the possibility [8] to test it experimentally at high-energy colliders. In the context of electroweak interactions, extra dimensions provide new mechanisms for symmetry breaking [9, 10] and the generation

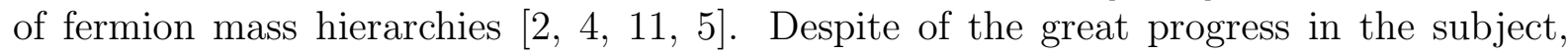
some relevant phenomenological and theoretical questions are still open. Among others, we have in mind the following general problem. Let $\left\{\Phi_{i}\right\}$ be a system of quantum fields propagating in a bulk space $\mathcal{M}$ with extra dimensions and with a nontrivial boundary $\partial \mathcal{M}$, representing our $3+1$ dimensional space-time (3-brane). The problem is to characterize the fields $\left\{\varphi_{i}\right\}$ induced by $\left\{\Phi_{i}\right\}$ on $\partial \mathcal{M}$. It is natural to expect (12, 13]) that the correspondence $\left\{\varphi_{i}\right\} \leftrightarrow\left\{\Phi_{i}\right\}$ represents an essential point in understanding the deep relation between bulk and brane dynamics. Since in passing from $\mathcal{M}$ to $\partial \mathcal{M}$ one is suppressing spatial dimensions, it is not surprising that local fields in the bulk give rise to local fields on the brane. For a complete understanding of the map $\left\{\varphi_{i}\right\} \leftrightarrow\left\{\Phi_{i}\right\}$ however, it is essential to know also whether there exist bulk fields, which in spite of being nonlocal in $\mathcal{M}$, induce local fields on the brane $\partial \mathcal{M}$. The physical relevance of this question stems from the observation that local commutativity, which is a crucial prerequisite on the brane, in principle may be violated in the bulk. The main goal of the present paper is to explore this possibility. We explicitly construct a class of nonlocal bulk fields $\left\{\Phi_{i}\right\}$ which induce local fields $\left\{\varphi_{i}\right\}$ on $\partial \mathcal{M}$, respecting all fundamental physical requirements like Poincaré invariance, spectral condition and positivity. We first investigate the characteristic features of $\left\{\varphi_{i}\right\}$, focusing afterwards on two distinguished subfamilies which generate renormalizable and conformal invariant brane theories respectively. We analyze also some related aspects concerning the interplay between bulk and brane symmetries, studying in particular brane vector currents induced by conserved currents in the bulk. We start by considering a flat bulk space $\mathcal{M}$ and then discuss the case when $\mathcal{M}$ is a

slice of anti-de-Sitter (AdS) space-time, which attracts much attention [14, 15, 16, 17] in relation with the Randall-Sundrum proposal [5, 6] for brane localization of gravity.

The paper is organized as follows. In the next section we describe the framework and construct a specific class of bulk fields and their brane relatives. We examine in detail the influence of the brane on the quantization, detecting and parametrizing the freedom left after imposing the boundary conditions. The central points are the analysis of locality 
and the issues of renormalizability and conformal invariance. In section 3 we establish a bridge between bulk and brane symmetries. Here we derive also Ward identities on the brane. Section 3 extends the formalism to an AdS background. The last section is devoted to our conclusions.

\section{Quantization in the presence of a brane}

The purpose of this section is to develop a general framework for studying quantum fields induced on branes. For a bulk space we take the manifold $\mathcal{M}=\mathbb{R}^{4} \times \mathbb{R}_{+}$, where $\mathbb{R}_{+}$is the half line $\{y \in \mathbb{R}: y>0\}$. It is convenient to adopt the coordinates $(x, y) \in \mathbb{R}^{4} \times \mathbb{R}_{+}$ where $x \equiv\left(x^{0}, x^{1}, x^{2}, x^{3}\right)=\left(x^{0}, \mathbf{x}\right)$. In this section $\mathcal{M}$ is equipped with the flat metric

$$
G_{\alpha \beta}=\left(\begin{array}{cc}
g & 0 \\
0 & -1
\end{array}\right) \quad, \quad \operatorname{diag} g=(1,-1,-1,-1), \quad \alpha, \beta=0, \ldots, 4 .
$$

The boundary $\partial M$ coincides with the $3+1$-dimensional Minkowski space $\mathbf{M}_{3+1} \equiv\left\{\mathbb{R}^{4}, g\right\}$, representing the 3 -brane.

In order to illustrate in its simplest form the general mechanism for inducing quantum fields on $\mathbf{M}_{3+1}$ from $\mathcal{M}=\mathbb{R}^{4} \times \mathbb{R}_{+}$, we consider a free Hermitian scalar field with mass $M \geq 0$. The corresponding action reads

$$
S_{0}=\frac{1}{2} \int_{-\infty}^{\infty} d^{4} x \int_{0}^{\infty} d y:\left(\partial^{\alpha} \Phi \partial_{\alpha} \Phi-M^{2} \Phi \Phi\right):(x, y)-\frac{\eta}{2} \int_{-\infty}^{\infty} d^{4} x: \Phi \Phi:(x, 0),
$$

where $\eta \in \mathbb{R}$ and : ... : indicates the normal product with respect to the creation and annihilation operators introduced few lines below. The variation of $S_{0}$ gives both the equation of motion

$$
\left(\partial^{\alpha} \partial_{\alpha}+M^{2}\right) \Phi(x, y)=0
$$

and the so called mixed boundary condition

$$
\lim _{y \downarrow 0}\left(\partial_{y}-\eta\right) \Phi(x, y)=0
$$

The parameter $\eta$ has dimension of a mass; in the limits $\eta \rightarrow 0$ and $\eta \rightarrow \infty$ one recovers from (团) the Neumann and Dirichlet boundary conditions respectively. Other boundary conditions [18 can be treated analogously. 
We quantize eqs.(3, (3) using the operator formalism, which allows a better control on locality and positivity then the functional integral approach. As already explained in [19], stability considerations imply the lower bound

$$
\eta \geq-M
$$

which is assumed throughout the paper. The field $\Phi$, satisfying eqs.(3,4), admits the decomposition

$$
\begin{gathered}
\Phi(x, y)=\int_{-\infty}^{\infty} \frac{d^{3} p}{(2 \pi)^{3}}\left\{b^{*}(\mathbf{p}) E_{M^{2}-\eta^{2}}(x, \mathbf{p}) \psi_{b}(y)+b(\mathbf{p}) \bar{E}_{M^{2}-\eta^{2}}(x, \mathbf{p}) \bar{\psi}_{b}(y)+\right. \\
\left.\int_{0}^{\infty} \frac{d \lambda}{2 \pi}\left[a^{*}(\mathbf{p}, \lambda) E_{M^{2}+\lambda^{2}}(x, \mathbf{p}) \psi(y, \lambda)+a(\mathbf{p}, \lambda) \bar{E}_{M^{2}+\lambda^{2}}(x, \mathbf{p}) \bar{\psi}(y, \lambda)\right]\right\},
\end{gathered}
$$

where

$$
\begin{gathered}
E_{m^{2}}(x, \mathbf{p})=\frac{1}{\sqrt{2 \omega_{m^{2}}(\mathbf{p})}} \mathrm{e}^{-i \omega_{m^{2}}(\mathbf{p}) x^{0}+i \mathbf{p x}}, \quad \omega_{m^{2}}(\mathbf{p})=\sqrt{\mathbf{p}^{2}+m^{2}} \\
\psi(y, \lambda)=\mathrm{e}^{-i \lambda y}+\frac{\lambda-i \eta}{\lambda+i \eta} \mathrm{e}^{i \lambda y}, \quad \psi_{b}(y)=\theta(-\eta) \sqrt{2|\eta|} \mathrm{e}^{\eta y}
\end{gathered}
$$

and the bar stands for complex conjugation. The basic ingredients of the superposition (6) are essentially two:

(i) the system of eigenfunctions (17,8);

(ii) the set $\left\{a^{*}(\mathbf{p}, \lambda), a(\mathbf{p}, \lambda), b^{*}(\mathbf{p}), b(\mathbf{p}): \mathbf{p} \in \mathbb{R}^{3}, \lambda \in \mathbb{R}_{+}\right\}$of creation and annihilation operators.

The functional input (i) is uniquely determined by the equation of motion (3) and the boundary condition (4). Besides the familiar plane waves $E_{m^{2}}(x, \mathbf{p})$, Eq.(6) involves the functions (8) related to the Hamiltonian operator $-\partial_{y}^{2}$ on $\mathbb{R}_{+}: \psi(y, \lambda)$ describe scattering states, whereas $\psi_{b}(y)$ is the unique bound state (with energy $-\eta^{2}$ ) present only for $\eta<0$. These functions form an orthonormal system and satisfy the completeness relation

$$
\int_{0}^{\infty} \frac{d \lambda}{2 \pi} \bar{\psi}\left(y_{1}, \lambda\right) \psi\left(y_{2}, \lambda\right)+\theta(-\eta) \psi_{b}\left(y_{1}\right) \psi_{b}\left(y_{2}\right)=\delta\left(y_{1}-y_{2}\right), \quad y_{1}, y_{2} \in \mathbb{R}_{+}
$$


with

$$
\theta(\alpha)= \begin{cases}1 & \text { if } \alpha>0 \\ 0 & \text { if } \alpha \leq 0\end{cases}
$$

Concerning the algebraic input (ii), there is certain freedom reflecting an intrinsic feature of the system under consideration, namely, the breakdown of bulk Poincaré invariance due to the brane. This freedom can be parametrized by a pair $\left(\sigma, \sigma_{b}\right)$, where $\sigma(\lambda)$ is a distribution on $[0, \infty)$ and $\sigma_{b} \in \mathbb{R}$. Any $\left(\sigma, \sigma_{b}\right)$ defines an algebra $\mathcal{A}_{\left(\sigma, \sigma_{b}\right)}$ generated by $\left\{a^{*}(\mathbf{p}, \lambda), a(\mathbf{p}, \lambda), b^{*}(\mathbf{p}), b(\mathbf{p})\right\}$ subject to the constraints:

$$
\begin{aligned}
& {\left[a\left(\mathbf{p}_{1}, \lambda_{1}\right), a^{*}\left(\mathbf{p}_{2}, \lambda_{2}\right)\right]=(2 \pi)^{4} \delta\left(\mathbf{p}_{1}-\mathbf{p}_{2}\right) \sigma\left(\lambda_{1}\right) \delta\left(\lambda_{1}-\lambda_{2}\right),} \\
& {\left[a\left(\mathbf{p}_{1}, \lambda_{1}\right), a\left(\mathbf{p}_{2}, \lambda_{2}\right)\right]=\left[a^{*}\left(\mathbf{p}_{1}, \lambda_{1}\right), a^{*}\left(\mathbf{p}_{2}, \lambda_{2}\right)\right]=0,} \\
& {\left[b\left(\mathbf{p}_{1}\right), b^{*}\left(\mathbf{p}_{2}\right)\right]=(2 \pi)^{3} \sigma_{b} \delta\left(\mathbf{p}_{1}-\mathbf{p}_{2}\right),} \\
& {\left[b\left(\mathbf{p}_{1}\right), b\left(\mathbf{p}_{2}\right)\right]=\left[b^{*}\left(\mathbf{p}_{1}\right), b^{*}\left(\mathbf{p}_{2}\right)\right]=0 .}
\end{aligned}
$$

The requirement of positivity of the metric in the Fock representations $\mathcal{F}\left(\mathcal{A}_{\left(\sigma, \sigma_{b}\right)}\right)$ of $\mathcal{A}_{\left(\sigma, \sigma_{b}\right)}$ implies the restrictions

$$
\sigma(\lambda) \geq 0, \quad \sigma_{b} \geq 0
$$

The data $\left(\sigma, \sigma_{b}\right)$ have a deep structural impact on different levels. We focus first on the equal-time $\Phi$-commutators generated by (11, 13). The nontrivial one is

$$
\begin{gathered}
{\left[\left(\partial_{0} \Phi\right)\left(x^{0}, \mathbf{x}_{1}, y_{1}\right), \Phi\left(x^{0}, \mathbf{x}_{2}, y_{2}\right)\right]=} \\
-i \delta\left(\mathbf{x}_{1}-\mathbf{x}_{2}\right)\left[\int_{0}^{\infty} \frac{d \lambda}{2 \pi} \bar{\psi}\left(y_{1}, \lambda\right) \sigma(\lambda) \psi\left(y_{2}, \lambda\right)+\theta(-\eta) \sigma_{b} \psi_{b}\left(y_{1}\right) \psi_{b}\left(y_{2}\right)\right]
\end{gathered}
$$

and represents a generalization of the standard canonical commutation relation. Because of (9), one recovers the latter for $\left(\sigma, \sigma_{b}\right)=(1,1)$. We therefore conclude that the pair $\left(\sigma, \sigma_{b}\right)$ parametrizes a class $\mathcal{S}$ of generalized canonical structures. Each of them defines an initial condition for the time evolution in the bulk, which is compatible with the bulk symmetries. It will be shown later on, that besides this bulk interpretation, $\left(\sigma, \sigma_{b}\right)$ has a clear physical meaning also from the brane view point.

Let us investigate now the locality properties of $\Phi$. One easily derives

$$
\begin{gathered}
{\left[\Phi\left(x_{1}, y_{1}\right), \Phi\left(x_{2}, y_{2}\right)\right]=-2 i \sigma_{b} \theta(-\eta)|\eta| \mathrm{e}^{\eta \widetilde{y}_{12}} D_{M^{2}-\eta^{2}}\left(x_{12}\right)+} \\
-i \int_{0}^{\infty} \frac{d \lambda}{2 \pi} \frac{4 \sigma(\lambda)}{\lambda^{2}+\eta^{2}}\left(\lambda \cos \lambda y_{1}+\eta \sin \lambda y_{1}\right)\left(\lambda \cos \lambda y_{2}+\eta \sin \lambda y_{2}\right) D_{M^{2}+\lambda^{2}}\left(x_{12}\right),
\end{gathered}
$$


where $x_{12}=x_{1}-x_{2}, \widetilde{y}_{12}=y_{1}+y_{2}$ and

$$
D_{m^{2}}(x)=i \int_{-\infty}^{\infty} \frac{d^{4} p}{(2 \pi)^{4}} \mathrm{e}^{-i p x}\left[\theta\left(p^{0}\right)-\theta\left(-p^{0}\right)\right] 2 \pi \delta\left(p^{2}-m^{2}\right), \quad p=\left(p^{0}, \mathbf{p}\right),
$$

is the well-known Pauli-Jordan function in $\mathbf{M}_{3+1}$. In (17) and in what follows, the integration in $\lambda$ is understood in the sense of distributions, i.e. after smearing with test functions in $\left(x_{1}, y_{1}\right)$ and $\left(x_{2}, y_{2}\right)$. One can demonstrate [19 that $\Phi$ is a local field for $\left(\sigma, \sigma_{b}\right)=(1,1)$. We observe in passing that this property is not so straightforward as on manifolds without boundaries, because one must take into account [20, 21] that signals propagating in the bulk are reflected from the brane as well. For generic $\left(\sigma, \sigma_{b}\right) \neq(1,1)$ the field $\Phi$ is nonlocal, i.e. there exist space-like separated points $\left(x_{1}, y_{1}\right)$ and $\left(x_{2}, y_{2}\right)$ in which the right hand side of eq.(17) does not vanish. Nevertheless, we will prove below that local commutativity is restored on the brane.

We have shown so far that even for a fixed boundary condition (何), the quantization of the action $S_{0}$ is not unique if the requirement of locality in the bulk is relaxed. Each pair $\left(\sigma, \sigma_{b}\right) \in \mathcal{S}$ defines by means of eqs.(6,11,13) a bulk field $\Phi$, which is in general nonlocal, though generated by a local bulk action. As already mentioned, the origin of this unusual feature is the defect, produced in the bulk by the brane. Being codified in the algebra $\mathcal{A}_{\left(\sigma, \sigma_{b}\right)}$, this is a genuine quantum phenomenon.

The two-point vacuum expectation value (Wightman function) of $\Phi$ in the Fock space $\mathcal{F}\left(\mathcal{A}_{\left(\sigma, \sigma_{b}\right)}\right)$ reads

$$
\begin{gathered}
\left\langle\Phi\left(x_{1}, y_{1}\right) \Phi\left(x_{2}, y_{2}\right)\right\rangle_{0}=2 \sigma_{b} \theta(-\eta)|\eta| \mathrm{e}^{\eta \widetilde{y}_{12}} W_{M^{2}-\eta^{2}}\left(x_{12}\right)+ \\
\int_{0}^{\infty} \frac{d \lambda}{2 \pi} \frac{4 \sigma(\lambda)}{\lambda^{2}+\eta^{2}}\left(\lambda \cos \lambda y_{1}+\eta \sin \lambda y_{1}\right)\left(\lambda \cos \lambda y_{2}+\eta \sin \lambda y_{2}\right) W_{M^{2}+\lambda^{2}}\left(x_{12}\right)
\end{gathered}
$$

where

$$
W_{m^{2}}(x)=\int_{-\infty}^{\infty} \frac{d^{4} p}{(2 \pi)^{4}} \mathrm{e}^{-i p x} \theta\left(p^{0}\right) 2 \pi \delta\left(p^{2}-m^{2}\right)
$$

is the two-point scalar function of mass $m^{2}$ in $\mathbf{M}_{3+1}$. Since $\Phi$ is a free field, eq.(19) completely fixes all of its correlation functions. The latter define via

$$
\left\langle\varphi\left(x_{1}\right) \cdots \varphi\left(x_{n}\right)\right\rangle_{0}=\lim _{y_{i} \downarrow 0}\left\langle\Phi\left(x_{1}, y_{1}\right) \cdots \Phi\left(x_{n}, y_{n}\right)\right\rangle_{0},
$$

the field $\varphi$ induced on the brane. The existence of the limit follows directly from eq. (19). The fundamental features of $\varphi$ are encoded in the two-point function

$$
\begin{gathered}
w\left(x_{12}\right)=\left\langle\varphi\left(x_{1}\right) \varphi\left(x_{2}\right)\right\rangle_{0}= \\
\int_{0}^{\infty} \frac{d \lambda}{2 \pi} \frac{4 \lambda^{2} \sigma(\lambda)}{\lambda^{2}+\eta^{2}} W_{M^{2}+\lambda^{2}}\left(x_{12}\right)+2 \sigma_{b} \theta(-\eta)|\eta| W_{M^{2}-\eta^{2}}\left(x_{12}\right) .
\end{gathered}
$$


An obvious change of variables leads to the Källén-Lehmann representation

$$
w\left(x_{12}\right)=\int_{0}^{\infty} d \lambda^{2} \varrho\left(\lambda^{2}\right) W_{\lambda^{2}}\left(x_{12}\right)
$$

with

$$
\varrho\left(\lambda^{2}\right)=\theta\left(\lambda^{2}-M^{2}\right) \frac{\sqrt{\lambda^{2}-M^{2}} \sigma\left(\sqrt{\lambda^{2}-M^{2}}\right)}{\pi\left(\lambda^{2}+\eta^{2}-M^{2}\right)}+2 \sigma_{b} \theta(-\eta)|\eta| \delta\left(M^{2}-\eta^{2}-\lambda^{2}\right),
$$

Therefore, $\varphi$ is a generalized free field, which can be fully reconstructed (see e.g. sect. II.6 of [22]) from the two-point function (22). The conditions (15) imply that $\varrho\left(\lambda^{2}\right) d \lambda^{2}$ is a positive measure on $\left[M^{2}, \infty\right)$, thus ensuring that the underlying state space is a Hilbert space. Brane Poincaré invariance is manifest.

The spectrum of the $\varphi$-mass operator belongs to $\left[M^{2}, \infty\right)$ and if $-M \leq \eta<0$ has an additional pure point contribution at $M^{2}-\eta^{2}$. The states contributing to $\left[M^{2}, \infty\right)$ represent the Kaluza-Klein (KK) modes, whereas $M^{2}-\eta^{2}$ is the mass of the state corresponding to $\psi_{b}$. In this context the pair $\sigma$ defines the weight by which each KK mode contributes to the field $\varphi$. This is actually the physical interpretation of $\sigma$ on the brane level. For $\eta=0$ and $\sigma(\lambda)=\pi \mu \delta\left(\lambda^{2}-\mu^{2}\right)$, the free field with mass $m^{2}=M^{2}+\mu^{2}$ is localized on the brane.

The evaluation of the integral in Eq. (23) is straightforward in momentum space. For the Fourier transform $\widehat{w}$ of $w$ one gets

$$
\widehat{w}(p)=2 \theta\left(p^{0}\right)\left[\theta\left(p^{2}-M^{2}\right) \frac{\sqrt{p^{2}-M^{2}} \sigma\left(\sqrt{p^{2}-M^{2}}\right)}{p^{2}-M^{2}+\eta^{2}}+\sigma_{b} \theta(-\eta)|\eta| 2 \pi \delta\left(p^{2}-M^{2}+\eta^{2}\right)\right],
$$

implying that for generic $\sigma$ the induced field $\varphi$ does not satisfy the free Klein-Gordon equation and cannot be derived from a local action (integral of a local density) on the brane. Nevertheless, $\varphi$ is a local field because

$$
\left[\varphi\left(x_{1}\right), \varphi\left(x_{2}\right)\right]=-i \int_{0}^{\infty} d \lambda^{2} \varrho\left(\lambda^{2}\right) D_{\lambda^{2}}\left(x_{12}\right) .
$$

Thus the limit $y \rightarrow 0$ absorbs all noncausal effects, regarding the behavior of $\Phi$. From (26) one gets

$$
\left[\left(\partial_{0} \varphi\right)\left(x^{0}, \mathbf{x}_{1}\right), \varphi\left(x^{0}, \mathbf{x}_{2}\right)\right]=-i \delta\left(\mathbf{x}_{1}-\mathbf{x}_{2}\right) \int_{0}^{\infty} d \lambda^{2} \varrho\left(\lambda^{2}\right)
$$


Therefore, if

$$
C \equiv \int_{0}^{\infty} d \lambda^{2} \varrho\left(\lambda^{2}\right)<\infty
$$

$\varphi$ satisfies the conventional canonical commutation relation up to a finite multiplicative field renormalization.

The bound (28) selects a subclass $\mathcal{S}_{\text {ren }} \subset \mathcal{S}$ of pairs $\left(\sigma, \sigma_{b}\right)$, generating brane fields with distinguished ultraviolet (UV) behavior. Indeed, from (26) one obtains for the propagator

$$
\tau\left(x_{12}\right)=i\left\langle T \varphi\left(x_{1}\right) \varphi\left(x_{2}\right)\right\rangle_{0}=\int_{0}^{\infty} d \lambda^{2} \varrho\left(\lambda^{2}\right) \Delta_{\lambda^{2}}\left(x_{12}\right),
$$

$T$ indicating time ordering and

$$
\Delta_{m^{2}}(x)=-\int_{-\infty}^{\infty} \frac{d^{4} p}{(2 \pi)^{4}} \frac{\mathrm{e}^{-i p x}}{p^{2}-m^{2}+i \varepsilon} .
$$

In momentum space one has

$$
\widehat{\tau}(p)=-\int_{0}^{\infty} d \lambda^{2} \varrho\left(\lambda^{2}\right) \frac{1}{p^{2}-\lambda^{2}+i \varepsilon}
$$

Combining (28) with (31), one gets the estimate

$$
p_{E}^{2} \widehat{\tau}\left(p_{E}\right) \leq C, \quad p_{E}=\left(-i p^{0}, \mathbf{p}\right) .
$$

Therefore, at large Euclidean momenta $p_{E}$ the $\varphi$-propagator decays at least like $1 / p_{E}^{2}$, provided that (28) is satisfied. This is not the case when (28) is violated. In fact, for the local (canonical) bulk field $\left(\sigma, \sigma_{b}\right)=(1,1)$, considered till now in the literature, one finds

$$
\widehat{\tau}_{(1,1)}(p)=-\frac{\sqrt{M^{2}-p^{2}}-\eta}{p^{2}-M^{2}+\eta^{2}+i \epsilon},
$$

which decays like $1 / \sqrt{p_{E}^{2}}$ when $p_{E}^{2} \rightarrow \infty$.

The above result can be used to construct renormalizable theories on the brane. The simplest example coming in mind is the model

$$
S=S_{0}+\frac{g}{3 !} \int_{-\infty}^{\infty} d^{4} x: \Phi^{3}:(x, 0) .
$$


Giving up local commutativity in the bulk implies that the quantization of (34) is not uniquely defined: one must choose $\left(\sigma, \sigma_{b}\right) \in \mathcal{S}$. If $\left(\sigma, \sigma_{b}\right) \in \mathcal{S}_{\text {ren }}$, one gets both a renormalizable and local theory on the brane. A concrete example is $\left(\sigma, \sigma_{b}\right)=\left(\mu^{2} / \lambda^{2}, \sigma_{b}\right)$, where $\mu$ is a parameter. Evaluating the integral (31), one gets

$$
\widehat{\tau}_{\left(\mu^{2} / \lambda^{2}, \sigma_{b}\right)}(p)=-\frac{1}{p^{2}-M^{2}+\eta^{2}+i \epsilon}\left[\frac{\mu^{2} \sqrt{M^{2}-p^{2}}}{p^{2}-M^{2}+i \epsilon}+\frac{\mu^{2}}{|\eta|}+2 \sigma_{b} \theta(-\eta)|\eta|\right],
$$

leading to a renormalizable perturbative expansion for the correlation functions of $\varphi$.

One may wonder if by a suitable choice of $\left(\sigma, \sigma_{b}\right)$ one cannot further improve the $1 / p_{E}^{2}$ UV-decay of (35), getting a perturbatively finite theory on the brane. We observe in this respect that the restrictions (15) imply the estimate

$$
\widehat{\tau}\left(p_{E}\right) \geq \frac{C^{\prime}(\Lambda)}{p_{E}^{2}+\Lambda^{2}}
$$

$\Lambda$ and $C^{\prime}(\Lambda)$ being some constants. Therefore, positivity of the metric in the state space prevents an UV-decay faster then $1 / p_{E}^{2}$. On the contrary, allowing for violations of (15), one can obtain UV-finite models on the brane. For instance, setting

$$
\sigma_{b}=-\frac{\mu^{2}}{2 \eta^{2}}, \quad \eta<0
$$

in eq. (35), one has UV-finite perturbative expansion for the model (34). This property can be used for developing an UV-regularization procedure on the brane.

We conclude this section with a discussion of brane conformal invariance. Let $M=$ $\eta=0$ and let us consider the one-parameter family

$$
\sigma(\lambda)=\frac{\pi}{\Gamma(d-1)} \lambda^{2 d-3}
$$

postponing for a while the justification of the normalization factor. We also require that $\sigma(\lambda)$ has at most integrable singularity in $\lambda=0$, namely

$$
d>1
$$

Since $\eta=0$, the value of $\sigma_{b}$ is irrelevant and inserting (38) in eq.(25), one finds

$$
\widehat{w}(p)=\frac{2 \pi}{\Gamma(d-1)} \theta\left(p^{0}\right) \theta\left(p^{2}\right)\left(p^{2}\right)^{d-2} \equiv \frac{2 \pi}{\Gamma(d-1)} \theta\left(p^{0}\right)\left(p^{2}\right)_{+}^{d-2},
$$


which is (see e.g. sect.IV.1 of [23]) the two-point function of a conformal covariant scalar field of dimension $d$, the lower bound (39) ensuring positivity. Therefore, our framework provides a mechanism for generating local conformal scalar fields on the brane of any dimension $d>1$. With the exception of $d=\frac{3}{2}$, all these fields are induced by nonlocal bulk fields, which confirms once more the relevance of giving up local commutativity in the bulk. By means of the identity

$$
\lim _{d \downarrow 1} \frac{1}{\Gamma(d-1)}\left(p^{2}\right)_{+}^{d-2}=\delta\left(p^{2}\right)
$$

which holds 24 in the sense of distributions, one recovers from (40) the free massless field in the limit $d \rightarrow 1$. This fact explains the choice of normalization in (38).

Summarizing, we described in this section the influence of a brane on the bulk quantization. We considered for illustration the case of $s=1$ noncompact extra dimensions, but the framework and the results have a direct generalization to $s \geq 1$. In that case $\lambda \mapsto\left(\lambda_{1}, \ldots, \lambda_{s}\right)$, where $\lambda_{i}$ will have discrete spectrum if the corresponding dimension is compact.

\section{Currents induced on a brane}

The aim of this section is to display a link between bulk and brane symmetries, establishing further interesting features of the class $\mathcal{S}_{\text {ren. }}$ We begin with some preliminary considerations on classical level. Let $J_{\alpha}$ be a conserved current in the bulk. With our choice of coordinates, this means that

$$
\partial^{\mu} J_{\mu}(x, y)-\partial_{y} J_{4}(x, y)=0, \quad \mu=0, \ldots, 3 .
$$

The corresponding charge

$$
Q=\int_{-\infty}^{\infty} d^{3} x \int_{0}^{\infty} d y J_{0}(x, y)
$$

is time-independent if $J_{\alpha}$ decay fast enough when both $|\mathbf{x}| \rightarrow \infty, y \rightarrow \infty$ and

$$
\int_{-\infty}^{\infty} d^{3} x J_{4}(x, 0)=0
$$

Eq.(44), which is a direct consequence of (42) and the Gauss divergence theorem, represents a kind of integral boundary condition with transparent physical interpretation: $Q$ 
is conserved if and only if the total flux of the bulk current across $\mathbb{R}^{3} \subset \mathbf{M}_{3+1}$ vanishes for any $x^{0}$. Because of (42), the boundary induced current

$$
j_{\mu}(x)=\lim _{y \downarrow 0} J_{\mu}(x, y),
$$

is not conserved in general. As suggested by eq.(42), in order to construct a conserved current in $\mathbf{M}_{3+1}$, we introduce the brane scalar field

$$
\chi(x)=\lim _{y \downarrow 0} \partial_{y} J_{4}(x, y),
$$

and define

$$
k_{\mu}(x)=j_{\mu}(x)-\left(\partial_{\mu} \Delta_{0} * \chi\right)(x),
$$

where $*$ denotes a convolution and $\Delta_{0}$ is defined by eq.(30). Now, eqs.(30,42,47) imply

$$
\partial^{\mu} k_{\mu}(x)=0,
$$

if

$$
\partial^{\mu} \lim _{y \downarrow 0} J_{\mu}(x, y)=\lim _{y \downarrow 0} \partial^{\mu} J_{\mu}(x, y) .
$$

Together with (44), the condition (49) is a basic requirement for the validity of our construction below.

Summarizing these classical considerations, we have seen that a conserved bulk current $J_{\alpha}$ induces both a vector current $j_{\mu}$ and a scalar field $\chi$ on the brane $\partial \mathcal{M}$. Generally, $j_{\mu}$ is not conserved. The improved current $k_{\mu}$ is conserved, but is expected to have worse localization properties, due to the convolution appearing in eq.(47).

We turn now to the quantum case, focusing on

$$
S_{0}=\int_{-\infty}^{\infty} d^{4} x \int_{0}^{\infty} d y:\left(\partial^{\alpha} \Phi^{*} \partial_{\alpha} \Phi-M^{2} \Phi^{*} \Phi\right):(x, y)-\eta \int_{-\infty}^{\infty} d^{4} x: \Phi^{*} \Phi:(x, 0),
$$

which describes a free complex scalar field

$$
\Phi(x, y)=\frac{1}{\sqrt{2}}\left[\Phi_{1}(x, y)+i \Phi_{2}(x, y)\right]
$$

whose real components $\Phi_{1}$ and $\Phi_{2}$ satisfy eqs.(33, (4). The invariance of (50) under global $U(1)$ transf ormations implies the conservation of the current

$$
J_{\alpha}(x, y)=i:\left[\Phi^{*}\left(\partial_{\alpha} \Phi\right)-\left(\partial_{\alpha} \Phi^{*}\right) \Phi\right]:(x, y) .
$$


Due to the boundary condition (田), one has

$$
\lim _{y \downarrow 0} J_{4}(x, y)=0, \quad \forall x \in \mathbf{M}_{3+1},
$$

which shows in turn that the current (52) satisfies (44). Though $J_{4}$ vanishes on the brane, we emphasize that its $y$-derivative $\chi$ does not.

Fixing the data $\left(\sigma, \sigma_{b}\right) \in \mathcal{S}$, we quantize both $\Phi_{1}$ and $\Phi_{2}$ according to the scheme developed in the previous section and are in position to compute any correlation function of the operators $J_{\alpha}, \Phi^{*}, \Phi$. The latter induce on the brane the fields $j_{\mu}, \chi, \varphi^{*}, \varphi$ by means of

$$
\begin{gathered}
\left\langle j_{\mu_{1}}\left(x_{1}\right) \cdots \chi\left(x_{k}\right) \cdots \varphi^{*}\left(x_{k+m+1}\right) \cdots \varphi\left(x_{k+m+n+1}\right) \cdots\right\rangle_{0}= \\
\lim _{y_{i} \downarrow 0}\left\langle J_{\mu_{1}}\left(x_{1}, y_{1}\right) \cdots \partial_{y_{k}} J_{4}\left(x_{k}, y_{k}\right) \cdots \Phi^{*}\left(x_{k+m+1}, y_{k+m+1}\right) \cdots \Phi\left(x_{k+m+n+1}, y_{k+m+n+1}\right) \cdots\right\rangle_{0} .
\end{gathered}
$$

It is clear from eq.(54) that $\varphi$ inherits the $U(1)$-symmetry from $\Phi$, which poses two main questions:

(a) does a conserved current, generating the brane $U(1)$-invariance, exist?

(b) in case of affirmative answer to (a), does this current satisfy a Ward identity?

The answers to (a) and (b) are encoded in the correlation functions (54). Let us consider the relevant ones, starting by

$$
\begin{gathered}
\left\langle j_{\mu}\left(x_{1}\right) j_{\nu}\left(x_{2}\right)\right\rangle_{0}= \\
2 \int_{0}^{\infty} d \lambda_{1}^{2} d \lambda_{2}^{2} \varrho\left(\lambda_{1}^{2}\right) \varrho\left(\lambda_{2}^{2}\right)\left[\left(\partial_{\mu} W_{\lambda_{1}^{2}}\right)\left(x_{12}\right)\left(\partial_{\nu} W_{\lambda_{2}^{2}}\right)\left(x_{12}\right)-\left(\partial_{\mu} \partial_{\nu} W_{\lambda_{1}^{2}}\right)\left(x_{12}\right) W_{\lambda_{2}^{2}}\left(x_{12}\right)\right] .
\end{gathered}
$$

Notice that the distribution product at coinciding points in the integrand of eq. (55) is well-defined and simply related to the product $W_{\lambda_{1}^{2}}(x) W_{\lambda_{2}^{2}}(x)$, whose Fourier transform

$$
\begin{gathered}
\int_{-\infty}^{\infty} \frac{d^{4} p}{(2 \pi)^{4}} \mathrm{e}^{-i p x} W_{\lambda_{1}^{2}}(x) W_{\lambda_{2}^{2}}(x)= \\
\frac{1}{8 \pi p^{2}} \theta\left(p^{0}\right) \theta\left(p^{2}-\left(\lambda_{1}+\lambda_{2}\right)^{2}\right) \sqrt{p^{2}-\left(\lambda_{1}+\lambda_{2}\right)^{2}} \sqrt{p^{2}-\left(\lambda_{1}-\lambda_{2}\right)^{2}}
\end{gathered}
$$


represents the phase space of two relativistic scalar particles of mass $\lambda_{1}^{2}$ and $\lambda_{2}^{2}$. Eq.(55) implies

$$
\left\langle\partial^{\mu} j_{\mu}\left(x_{1}\right) \partial^{\nu} j_{\nu}\left(x_{2}\right)\right\rangle_{0}=\int_{0}^{\infty} d \lambda_{1}^{2} d \lambda_{2}^{2} \varrho\left(\lambda_{1}^{2}\right) \varrho\left(\lambda_{2}^{2}\right)\left(\lambda_{1}^{2}-\lambda_{2}^{2}\right)^{2} W_{\lambda_{1}^{2}}\left(x_{12}\right) W_{\lambda_{2}^{2}}\left(x_{12}\right),
$$

which confirms that $j_{\mu}$ is not conserved. In fact, one can deduce from the correlators (54) that

$$
\partial^{\mu} j_{\mu}(x)=\chi(x)
$$

Therefore, the improved quantum current $k_{\mu}$ is conserved. In order to demonstrate that it is precisely $k_{\mu}$ which generates the brane $U(1)$ symmetry, one must verify the corresponding Ward identities. For this purpose we consider the vertex functions

$$
\begin{gathered}
\left\langle T j_{\mu}\left(x_{1}\right) \varphi^{*}\left(x_{2}\right) \varphi\left(x_{3}\right)\right\rangle_{0}= \\
i \int_{0}^{\infty} d \lambda_{1}^{2} d \lambda_{2}^{2} \varrho\left(\lambda_{1}^{2}\right) \varrho\left(\lambda_{2}^{2}\right)\left[\left(\partial_{\mu} \Delta_{\lambda_{1}^{2}}\right)\left(x_{12}\right) \Delta_{\lambda_{2}^{2}}\left(x_{13}\right)-\Delta_{\lambda_{1}^{2}}\left(x_{12}\right)\left(\partial_{\mu} \Delta_{\lambda_{2}^{2}}\right)\left(x_{13}\right)\right] \\
\left\langle T \chi\left(x_{1}\right) \varphi^{*}\left(x_{2}\right) \varphi\left(x_{3}\right)\right\rangle_{0}=i \int_{0}^{\infty} d \lambda_{1}^{2} d \lambda_{2}^{2} \varrho\left(\lambda_{1}^{2}\right) \varrho\left(\lambda_{2}^{2}\right)\left(\lambda_{2}^{2}-\lambda_{1}^{2}\right) \Delta_{\lambda_{1}^{2}}\left(x_{12}\right) \Delta_{\lambda_{2}^{2}}\left(x_{13}\right) .
\end{gathered}
$$

Combining eqs. (58-60) one gets

$$
\partial^{\mu}\left\langle T k_{\mu}\left(x_{1}\right) \varphi^{*}\left(x_{2}\right) \varphi\left(x_{3}\right)\right\rangle_{0}=i C \delta\left(x_{12}\right)\left\langle T \varphi^{*}\left(x_{2}\right) \varphi\left(x_{3}\right)\right\rangle_{0}-i C \delta\left(x_{13}\right)\left\langle T \varphi^{*}\left(x_{3}\right) \varphi\left(x_{2}\right)\right\rangle_{0}
$$

which is the conventional Ward identity, provided that the bound (28) holds. Otherwise, the right hand side of (61) diverges, which demonstrates the special status of the brane fields corresponding to $\mathcal{S}_{\text {ren }}$. We emphasize that the above analysis takes into account not only the zero mode, but the whole KK-tower as well. The problem of Ward identities in the AdS/CFT framework has been faced in [25].

In conclusion, a conserved current $J_{\alpha}$ in the bulk induces both a vector current $j_{\mu}$ and scalar $\chi$, the latter being essential in constructing a conserved brane current. We expect therefore the $\chi$-like degrees of freedom to be fundamental in model building with extra space dimensions. 


\section{Anti-de Sitter bulk space}

In this section we keep the bulk manifold $\mathcal{M}=\mathbb{R}^{4} \times \mathbb{R}_{+}$, but now equipped with the AdS metric

$$
G_{\alpha \beta}=\left(\begin{array}{cc}
g \mathrm{e}^{-2 a y} & 0 \\
0 & -1
\end{array}\right), \quad a>0
$$

Thus $\mathcal{M}$ is a slice of the five-dimensional AdS space-time, whose boundary $\partial \mathcal{M}$ still coincides with $\mathbf{M}_{3+1}$. The problem is to construct and investigate the scalar quantum field $\Phi$ defined by

$$
S_{0}=\frac{1}{2} \int_{-\infty}^{\infty} d^{4} x \int_{0}^{\infty} d y \sqrt{G}:\left(\partial^{\alpha} \Phi \partial_{\alpha} \Phi-M^{2} \Phi \Phi\right):(x, y)-\frac{\eta}{2} \int_{-\infty}^{\infty} d^{4} x \sqrt{g}: \Phi \Phi:(x, 0) .
$$

From (63) one infers the equation of motion

$$
\left(\partial^{\mu} \partial_{\mu}-\mathrm{e}^{2 a y} \partial_{y} \mathrm{e}^{-4 a y} \partial_{y}+\mathrm{e}^{-2 a y} M^{2}\right) \Phi(x, y)=0
$$

and the boundary condition (4). The quantization of $\Phi$ follows the scheme developed in section 2. A modification of the functional input (i) is required by the fact that instead of the Hamiltonian $-\partial_{y}^{2}$, one has now

$$
-\mathrm{e}^{2 a y} \partial_{y} \mathrm{e}^{-4 a y} \partial_{y}+\mathrm{e}^{-2 a y} M^{2}
$$

The operator (65) defines a well-known [26, 27] singular boundary value problem on $\mathbb{R}_{+}$, related to Bessel's equation. Setting

$$
\nu=\sqrt{4+\frac{M^{2}}{a^{2}}}, \quad \eta_{b}=(2-\nu) a,
$$

one has [19]:

$$
\begin{gathered}
\psi(y, \lambda)=\mathrm{e}^{2 a y}\left[J_{\nu}\left(\lambda a^{-1} \mathrm{e}^{a y}\right) \widetilde{Y}_{\nu}\left(\lambda a^{-1}\right)-Y_{\nu}\left(\lambda a^{-1} \mathrm{e}^{a y}\right) \widetilde{J}_{\nu}\left(\lambda a^{-1}\right)\right], \quad \lambda \in \mathbb{R}_{+}, \\
\psi_{b}(y)=\delta_{\eta \eta_{b}} \sqrt{2 a(\nu-1)} \mathrm{e}^{\eta_{b} y}
\end{gathered}
$$

with

$$
\widetilde{Z}_{\nu}(\zeta)=\frac{1}{2 \sqrt{1+\widetilde{\eta}^{2}}}\left[(1-2 \widetilde{\eta}) Z_{\nu}(\zeta)+2 \zeta Z_{\nu}^{\prime}(\zeta)\right], \quad \widetilde{\eta}=\frac{\eta}{a}-\frac{3}{2}
$$


$Z_{\nu}$ denoting the Bessel function $J_{\nu}$ or $Y_{\nu}$ of first and second kind respectively. Instead of (9), now the completeness relation is

$$
\int_{0}^{\infty} d \lambda \mu(\lambda) \bar{\psi}\left(y_{1}, \lambda\right) \psi\left(y_{2}, \lambda\right)+\delta_{\eta \eta_{b}} \psi_{b}\left(y_{1}\right) \psi_{b}\left(y_{2}\right)=\mathrm{e}^{2 a y_{1}} \delta\left(y_{12}\right)
$$

where

$$
\mu(\lambda)=\frac{\lambda a^{-1}}{\widetilde{J}_{\nu}^{2}\left(\lambda a^{-1}\right)+\widetilde{Y}_{\nu}^{2}\left(\lambda a^{-1}\right)} .
$$

The algebraic input (ii) must be also slightly modified: the creation and annihilation operators $\left\{a^{*}(\mathbf{p}, \lambda), a(\mathbf{p}, \lambda), b^{*}(\mathbf{p}), b(\mathbf{p})\right\}$ satisfy eqs.(11-14) with the replacement

$$
2 \pi \delta\left(\lambda_{1}-\lambda_{2}\right) \longmapsto \frac{1}{\mu\left(\lambda_{1}\right)} \delta\left(\lambda_{1}-\lambda_{2}\right) .
$$

The quantum field $\Phi$ in the AdS background is then given by eq.([) with the substitutions

$d \lambda \mapsto 2 \pi \mu(\lambda) d \lambda$ and (67,68). The field $\varphi$ induced on the brane is fully determined by its two-point function

$$
w\left(x_{12}\right)=\left\langle\varphi\left(x_{1}\right) \varphi\left(x_{2}\right)\right\rangle_{0}=\int_{0}^{\infty} d \lambda^{2} \varrho_{\mathrm{AdS}}\left(\lambda^{2}\right) W_{\lambda^{2}}\left(x_{12}\right),
$$

where

$$
\varrho_{\mathrm{AdS}}\left(\lambda^{2}\right)=\theta\left(\lambda^{2}\right) \frac{2 \mu\left(\sqrt{\lambda^{2}}\right) \sigma\left(\sqrt{\lambda^{2}}\right)}{\pi^{2}\left(1+\widetilde{\eta}^{2}\right) \sqrt{\lambda^{2}}}+2 \sigma_{b} \delta_{\eta \eta_{b}} a(\nu-1) \delta\left(\lambda^{2}\right) .
$$

As expected, the Källén-Lehmann measure $\varrho_{\text {AdS }}\left(\lambda^{2}\right) d \lambda^{2}$ keeps trace of $\left(\sigma, \sigma_{b}\right) \in \mathcal{S}$. Due to the interaction of $\Phi$ with the AdS background, there is in general no mass gap on the brane even for $M \neq 0$. Bearing in mind this novelty with respect to the flat case, the results of section 2 apply also to the slice of AdS space-time, considered above.

\section{Conclusions}

We have studied above quantum field theory on a $4+1$ dimensional bulk manifold with boundary - a 3-brane which represents the observable 3+1 dimensional space-time. The brane breaks the bulk Lorentz symmetry down to brane Lorentz symmetry and allows 
for nonlocal initial conditions compatible with the bulk symmetries. Technically, these conditions are parametrized by $\left(\sigma, \sigma_{b}\right) \in \mathcal{S}$. Each pair $\left(\sigma, \sigma_{b}\right)$ defines a generalized canonical commutation structure and provides a direct relation between the initial conditions for the time evolution in the bulk and the KK spectral measure on the brane. We have constructed the bulk fields associated with $\mathcal{S}$ and their brane relatives, whose basic properties are captured by the measure of the Källén-Lehmann representation of the two-point function. From the latter we have inferred the existence of a subset $\mathcal{S}_{\text {ren }} \subset \mathcal{S}$, generating renormalizable models on the brane. In this context we have investigated also the interplay between conserved bulk and brane currents and the validity of brane Ward identities. Our analysis covers the full spectrum of bulk excitations and sheds new light on the correspondence $\left\{\varphi_{i}\right\} \leftrightarrow\left\{\Phi_{i}\right\}$ between brane and bulk quantum fields, showing that nonlocal theories in $\mathcal{M}$ may induce local theories on $\partial \mathcal{M}$. The results, obtained initially in flat bulk space, are extended to the case of AdS background as well.

The freedom in the choice of initial conditions for the bulk quantization, discussed in this work, is an universal intrinsic feature of quantum field theory with extra dimensions, which must be taken in consideration. It can be used for improving the UV behavior of brane interactions and for inducing conformal covariant fields on the brane. We expect that nonlocal bulk fields will help for avoiding some no-go theorems [28] for the construction of Randall-Sundrum compactifications from supergravity. At the same time, the possibility to adopt various initial conditions $\left(\sigma, \sigma_{b}\right) \in \mathcal{S}$ reflects an ambiguity, which is not fixed by quantum field theory itself. In order to obtain some information about $\left(\sigma, \sigma_{b}\right)$, one has to resort most probably to a more fundamental setting like string theory. Clarifying this point represents an interesting open problem for further investigations.

\section{References}

[1] Polchinski J 1999 String Theory, vol.2 (Cambridge: Cambridge Univ. Press)

[2] Arkani-Hamed N, Dimopoulos S and Dvali G 1998 Phys. Lett. B 429263

[3] Arkani-Hamed N, Dimopoulos S and Dvali G 1999 Phys. Rev. D 59860

[4] Antoniadis I, Arkani-Hamed N, Dimopoulos S and Dvali G 1998 Phys. Lett. B 436 257

[5] Randall L and Sundrum R 1999 Phys. Rev. Lett. 833370 
[6] Randall L and Sundrum R 1999 Phys. Rev. Lett. 834690

[7] Rubakov V A and Shaposhnikov N E 1983 Phys. Lett. B 125136

[8] Giudice G F, Rattazzi R and Wells J D 1999 Nucl. Phys. B 5443

[9] Antoniadis I 1990 Phys. Lett. B 246377

[10] Arkani-Hamed N, Hall L, Nomura Y, Smith D and Weiner N 2001 Finite radiative electroweak symmetry breaking from the bulk Preprint hep-ph/0102090

[11] Arkani-Hamed N and Schmaltz M 2000 Phys. Rev. D 61033005

[12] 't Hooft G 1993 Dimensional reduction in quantum gravity Preprint gr-qc/9310026

[13] Susskind L 1995 J. Math. Phys. 366377

[14] Verlinde H 2000 Nucl. Phys. B 580264

[15] Anchordoqui L, Nunez C and Olsen K 2000 JHEP 1050

[16] Arkani-Hamed N, Porrati M and Randall L 2001 Holography and phenomenology Preprint hep-th/0012148

[17] Rattazzi R and Zaffaroni A 2001 Comments on the holographic picture of the Randall-Sundrum model Preprint hep-th/0012248

[18] Gilkey P B, Kirsten K and Vassilevich D V 2001 Heat trace asymptotics with transmittal boundary conditions and quantum brane-world scenario Preprint hepth/0101105.

[19] Mintchev M and Pilo L 2001 Nucl. Phys. B 592 219, hep-th/0007002

[20] Liguori A, Mintchev M and Zhao L 1998 Commun. Matyh. Phys. 194569

[21] Liguori A and Mintchev M 1998 Nucl. Phys. B 522345

[22] Jost R 1965 The General Theory of Quantized Fields (Providence, R.I: American Math. Soc.)

[23] Todorov I T, Mintchev M and Petkova V 1978 Conformal Invariance in Quantum Field Theory (Pisa: ETS-Scuola Normale Superiore) 
[24] Gel'fand I M and Shilov G E 1964 Generalized Functions, vol. 1 (New York: Academic Press)

[25] Corley S 2000 Phys. Lett. B 484141

[26] Titchmarsh E C 1946 Eigenfunction Expansions Associated with Second-order Differential Equations (Oxford: Clarendon press)

[27] Naimark M A 1967 Linear Differential Operators, Vol. 1-2 (London: G.G. Harrap and Co.)

[28] Maldacena J and Nunez C 2000 Supergravity description of field theories on curved manifolds and a no go theorem Preprint hep-th/0007018 